\title{
Fuzzy Linguistic in Geometric Modeling
}

Mohd Sallehuddin Husain, Abd Fatah Wahab, R.U.Gobithasaan

School of Informatics and Applied Mathematics, Universiti Malaysia Terengganu, 21300 Kuala Terengganu, Malaysia.

*Corresponding Author: salleh_85s@yahoo.com

\section{Article history :}

Received 5 October 2014

Accepted 27 February 2015

\section{GRAPHICAL ABSTRACT}

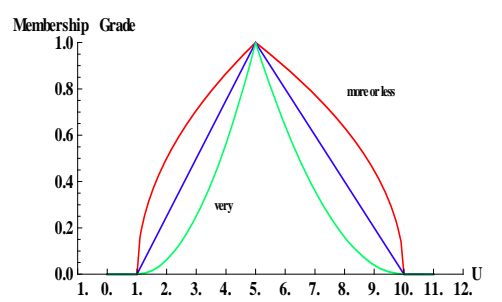

\section{ABSTRACT}

Fuzzy Linguistic is an extension of fuzzy set theory was introduced by Zadeh. Normally fuzzy linguistic is often associated with linguistic variables generated by a function modifier is also known as a hedges. This paper discusses the theorems and definitions of fuzzy linguistic perspective geometric modeling to produce Fuzzy Linguistic Control Point (FLCP). Fuzzy Linguistic Control Points have been blended in with the spline basic functions of the model to produce a few splines's model are characterized by fuzzy linguistics. At the end of this article will discuss some numerical examples of Fuzzy Linguistic Bezier Model.

Keywords: Fuzzy Linguistics, Fuzzy Linguistics Control Points, Fuzzy Linguistics Bezier Model

(C) 2015 Penerbit UTM Press. All rights reserved http://dx.doi.org/10.11113/mjfas.v11n1.344

\section{INTRODUCTION}

What is linguistics? Linguistics is the language used in everyday speech to describe an instruction and information. Moreover, linguistics also serves to interpret and analyze any data or information, help in decision making. Linguistics may exist in the form of oral (verbal) and non-verbal. Linguistics is very important in the lives of creatures in this world that all things and daily activities run smoothly and harmoniously according to its own understanding of language and dialect. Each linguistic or language used a different meaning depending on the individual's perception of itself. This is because the use of linguistic have a vary interpretation and assumptions. For example, the intonation of the words used in spoken Mandarin different meanings according to the spoken intonation. Likewise reading autobiographical verses in the Quran to mean different in intonation and 'counts' is read. Therefore linguistics is often associated with the uncertainty inherent in the concept of fuzzy sets.

Fuzzy set theory is the conclusion of the classical set theory was introduced by Lotfi A. Zadeh in 1965 [15]. Fuzzy set theory is a mathematical model presenting ambiguity or uncertainty that occur in everyday life. Through the fuzzy sets we can use fuzzy data, fuzzy rules and fuzzy information to help us make decisions about a situation or problem. Fuzzy set is characterized by a function in the interval $[0,1]$ that give value and the definition of membership in each set. The one in the set is an element of the set and the value 0 is not an element of the set. While a value between 0 and 1 are the fuzzy membership degree of the element in the set given. Each fuzzy value in the interval $[0,1]$ represents a particular purpose according to the degree of membership in the function.
Based on the definition above, this paper discusses the fuzzy linguistic that use the fuzzy hedges concept or modifier function in the fuzzy sets theory. In this paper, we discuss a new concept of fuzzy linguistic to be used in geometric modeling. We will define the Fuzzy Linguistic Coordinates as a Fuzzy Linguistic Control Point as a linguistic feature geometry coefficient. At the end of this paper discusses the use of Fuzzy Linguistic Control Point in modeling to produce a model of Fuzzy Linguistics Bezier.

\section{PRELIMINARIES}

Definition 1: (Fuzzy Set) Assume that $X$ is not the empty set. A fuzzy set $A$ in $X$ is characterized by a membership function $\mu_{A}: X \rightarrow[0,1]$ and $\mu_{A}(x)$ is called the degree of membership of element $x$ in fuzzy set $A$ for $x \in X$. Fuzzy sets are also a set of ordered pairs and can be written as $\tilde{A}=\left\{x, \mu_{A}(x): x \in X\right\}[5]$.

If $X=\left\{x_{1}, x_{2}, \ldots, x_{n}\right.$ is a finite set and $\tilde{A} \subseteq X$, then the fuzzy set $A$ can be written as

$\tilde{A}=\left\{\left(x_{1}, \mu_{A}\left(x_{1}\right)\right),\left(x_{2}, \mu_{A}\left(x_{2}\right)\right), \ldots,\left(x_{n}, \mu_{A}\left(x_{n}\right)\right)\right\} \subseteq X$

If $\tilde{A}$ and $\tilde{B}$ are two fuzzy sets in $X$, then the operations of intersection and union of fuzzy sets $A$ and $B$ in $X$ is defined as follows

$$
\begin{aligned}
& (\tilde{A} \cap \tilde{B})(x)=\min (\tilde{A}(x), \tilde{B}(x)) \\
& (\tilde{A} \cup \tilde{B})(x)=\operatorname{maks}(\tilde{A}(x), \tilde{B}(x))
\end{aligned}
$$

If $\tilde{A}$ and $\tilde{B}$ are represented in linguistic form, then $\tilde{A} \cap \tilde{B}$ and $\tilde{A} \cup \tilde{B}$ respectively translated as " $\tilde{A}$ and $\tilde{B}$ " and " $\tilde{A}$ or $\tilde{B}^{\prime \prime}$. Furthermore, the 
$\tilde{A} \subseteq \tilde{B} \Leftrightarrow(\forall x \in X)(\tilde{A}(x) \leq \tilde{B}(x))$

Definition 2: ( $\alpha$-cut) Assume that the fuzzy set $A$ (denoted $A_{\alpha}$ ) is a non-empty set. $A_{\alpha}$ have the degrees of membership $\mu_{A}(x)$ is defined as $A_{\alpha}=$ $\left\{x \in X \mid \mu_{A}(x) \geq \alpha\right\}$.

Definition 3: (Fuzzy Number) A fuzzy set $A$ is said to be a fuzzy number $A$ if $A$ on the real line are normal, convex, continuous, the membership function in the closed interval and the support $(A)=\left\{x \mid \mu_{A}(x)>0\right\}$ is limited [5].

Definition 4: (Trapezoidal Fuzzy Number) A fuzzy number $\tilde{A}=(a, b, c, d)$ is said to be a trapezoidal fuzzy number if its membership function is $[1,10]$

$\mu_{\tilde{A}}(x)=\left\{\begin{array}{cc}0, & x<a \\ \frac{x-a}{b-a}, & a<x \leq b \\ \frac{d-x}{d-c}, & b<x<c \\ 0, & x>d\end{array}\right.$

Definition 5: If $b=c$, the trapezoidal fuzzy numbers are defined as triangular fuzzy numbers, and can be written as $\tilde{A}=(a, b, d)$ By this point the triangular fuzzy number is a special case of trapezoidal fuzzy numbers [10.21].

\section{LINGUISTIC VARIABLES}

Linguistic variable was introduced by Zadeh in 1970. According to $[17,18]$ linguistic variables can be determined mathematically that linguistics variable is a quintuple $(x, T(x), U, G, M)$ where

$x$ is a name of variable;

$T(x)$ is the set of terms of the variables $x$ (set of names of linguistic values of $x$ );

$U$ is set the universe;

$G$ is the syntactical rules for generating $X$ for the $x$ (known as a term);

$M$ is a semantic rule for associating meaning $M(X)$ for each $X$

In the concept of linguistic variables, each variable has a specific set of terms variables generated by the function modifier known as hedges [6,9,16,23]. Each term variable is also known as a fuzzy linguistic. This fuzzy linguistic determined based on the strength modifier for membership functions in a fuzzy set that seeks to change the original terms of a value corresponding to the fuzzy set of linguistic values expressed in linguistic forms such as "very", "approximately", "too", "little" and so on. namely:

Fuzzy linguistic terms consist of two parts,

1) Fuzzy predicate such as young, old, good, deep, and so on.

2) Fuzzy modifier as 'very', 'more or less', 'very' and others.
Fuzzy modifier [11] is also known as hedges obscure acts to change the purpose of generating a new term for the predicate or key terms in a fuzzy set. Fuzzy hedges have two main properties to reflect the values of a fuzzy linguistic which is reinforcing and weakening.

\section{REINFORCING AND WEAKENING MODIFIER}

The reinforcing modifier gives new properties to the primary terms that are stronger than the original terms. Zadeh $[8,16,22]$ have introduced the modifier "very" as a mathematical interpretation to multiply two values of the membership function in the interval $[0,1]$ :

$\operatorname{very}(A)[x]=\mu_{A(x)}^{2}$

The weakening modifier [8,16,22] should give weak nature of the primary terms of the original terms as "more or less, less, less" and so on. Zadeh introduced the modifier "more or less" as a mathematical interpretation of the square root of the membership function in the interval $[0,1]$ See Figure 1.

more or $\operatorname{less}(A)[x]=\mu_{A(x)}^{0.5}$

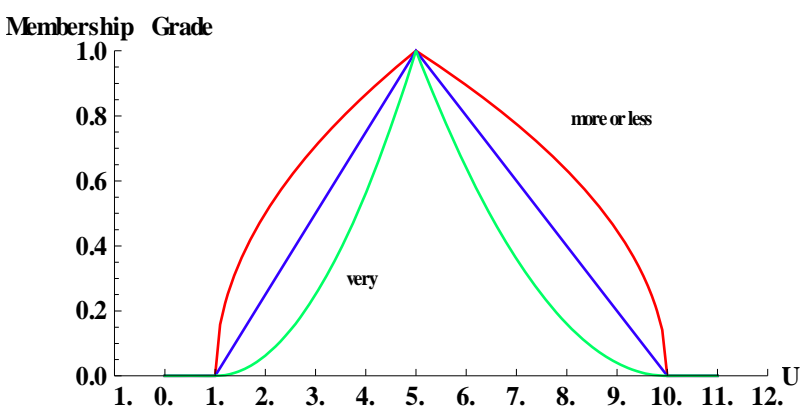

Figure 1. Examples of modifiers strong and weak modifiers in fuzzy hedge

\section{FUZZY LINGUISTIC IN GEOMETRIC MODELING}

In geometric modeling, data points obtained is defined as the control points [5.24]. The control points is known as a control polygon that's a geometric entities for splines. The control points were blended with a spline basis functions to produce spline models such as Bezier, B-splines and NURBS. However, since the data points are acquired is the uncertainty, a number of studies related to the geometry of the fuzzy model have been carried out $[2,3,4,7,12,13,19,24]$.

However, this paper discusses the geometric model of a fuzzy linguistic. We will introduce a new definition of fuzzy control points based on the concept of linguistic hedges which are found in the fuzzy sets theory. 
Proposition 1: Assume that $\tilde{L}$ is a triangular fuzzy set on the real line $R, \tilde{L}$ can be given as a set of nested intervals with $\alpha$-cut:

$[\tilde{L}]_{\alpha} \in \tilde{L}, \alpha \in[0,1]$

$L=\left\langle l_{\alpha}^{-}, l_{\alpha}, l_{\alpha}^{+}\right\rangle$is a support of $\tilde{L}$

where $l_{\alpha}^{-}, l_{\alpha}$ and $l_{\alpha}^{+}$are left fuzzy point, crisp point and right fuzzy points respectively.

$[\tilde{L}]_{1} \subset[\tilde{L}]_{\alpha_{n+2}} \subset[\tilde{L}]_{\alpha_{n+1}} \subset[\tilde{L}]_{\alpha_{n}} \subset[\tilde{L}]_{0}$

$\alpha_{n}<\alpha_{n+1} \Leftrightarrow[\tilde{L}]_{\alpha_{n+1}} \subset[\tilde{L}]_{\alpha_{n}}$

where $\alpha_{n} \in[0,1]$ and $n=0,1,2, \ldots, m$

Proposition 2: Suppose that $[\tilde{L}]_{\alpha}=\left(l_{1}, l_{2}, l_{3}, l_{4}\right)_{\alpha}$ and $[\tilde{R}]_{\alpha}=\left(r_{1}, r_{2}, r_{3}, r_{4}\right)_{\alpha}$ are two trapezoidal fuzzy numbers in any real space $R$ and $\alpha$ is the membership function in the interval $[0,1]$. If $[\tilde{L}]_{\alpha}<[\tilde{R}]_{\alpha}$, then $l<r$.

Propositiont 3: Let $[\tilde{L}]_{\alpha}$ and $[\tilde{R}]_{\alpha}$ are two fuzzy sets with fuzzy modifier with $\alpha$ is a $\alpha$-cut in $[0,1]$. Then $[\tilde{L}]_{\alpha}$ and $[\tilde{R}]_{\alpha}$ called fuzzy linguistic variables.

Proposition 4: Suppose $[\tilde{L}]_{\alpha^{k}}$ is a fuzzy linguistic variables on the real line $R$. If $\mu_{[\tilde{L}]_{\alpha^{k}}}=\alpha$, then $\alpha^{k} \subset \alpha$ with $k>0$.

Lemma 1: If $k=1$, then the membership function $\mu_{[\tilde{L}]_{\alpha} k}$ is linear and no hedge changes.

Lemma 2: Based on the equation (6), if $k=2$, the membership function $\mu_{[\widetilde{L}]_{\alpha^{k}}}$ is reinforcing modifier, then $\operatorname{very}[\widetilde{L}]=\alpha^{2}$

Lemma 3: Based on the equation (7), if $k=1 / 2$, the membership function $\mu_{[\tilde{L}]_{\alpha^{k}}}$ is weakening modifier, then more or $\operatorname{less}[\tilde{L}]=\alpha^{1 / 2}$

Corollary 1: Based on the Proposition 4 and Lemma (2)-(3). If $[\tilde{L}]_{\alpha}$ are fuzzy linguistic variables for "left", then $[\tilde{L}]_{\alpha^{2}}=$ veryleft and $[\tilde{L}]_{\alpha^{1 / 2}}=$ more or less left.

Corollary 2: Similarly, if $[\tilde{R}]_{\alpha}$ are fuzzy linguistic variables for the "right", based on the Proposition 4 and Lemma (2) - (3). Then $[\tilde{R}]_{\alpha^{2}}=$ very right and $[\tilde{R}]_{\alpha^{1 / 2}}=$ more or less right.

Theorem 1: Suppose that $[\tilde{L}]_{\alpha}$ and $[\tilde{R}]_{\alpha}$ are two fuzzy linguistic variables for "left" and "right" in real space $x$ axis and has a new linguistic terms. $[\tilde{L}]_{\alpha}$ and $[\tilde{R}]_{\alpha}$ be marked as

$[\tilde{L}]_{\alpha}=\left\{s(l)_{\alpha},(l)_{\alpha}, l b(l)_{\alpha}\right\}$

and
$[\tilde{R}]_{\alpha}=\left\{s(r)_{\alpha},(r)_{\alpha}, l b(r)_{\alpha}\right\}$

Equation (12) and (13) can be rewritten as

$[\text { left }]_{\alpha}=\left\{\right.$ very left $t_{\alpha}$, left $t_{\alpha}$, more or les left $\}$

$[\text { right }]_{\alpha}=\left\{\right.$ very right ${ }_{\alpha}$, right $_{\alpha}$, more or les right $\}$

Since $[\tilde{L}]_{\alpha}$ and $[\tilde{R}]_{\alpha}$ are two fuzzy linguistic variables on the same plane of the $x$-axis. The operations of intersection and union of fuzzy linguistic variables can occur as equation (2) and (3).

Example 1: Assume that $[\tilde{L}]_{\alpha}=\left\{s(l)_{\alpha},(l)_{\alpha}, l b(l)_{\alpha}\right\}$ and $[\tilde{R}]_{\alpha}=\left\{s(r)_{\alpha},(r)_{\alpha}, l b(r)_{\alpha}\right\}$ are left and right fuzzy linguistic variables on the same plane. The union and intersection operations are as follows:

a) Union operation of fuzzy linguistic variables

$[\tilde{L}]_{\alpha} \cup[\tilde{R}]_{\alpha}=\operatorname{Max}\left\{[\tilde{L}]_{\alpha},[\tilde{R}]_{\alpha}\right\}$

Proof:

$[\tilde{L}]_{\alpha} \cup[\tilde{R}]_{\alpha}=[\text { left }]_{\alpha}$ OR $[\text { right }]_{\alpha}$

(Associative Law)

$=(\text { very left }, \text { left }, \text { more or less left })_{\alpha}$

$\mathrm{OR}$ (very right, right, lmore or less right $)_{\alpha}$

$=\left\langle\left(\right.\right.$ very left $_{\alpha}$ OR very right $\left.{ }_{\alpha}\right)$,

(very left ${ }_{\alpha} O R$ right $_{\alpha}$ ),

(very left $t_{\alpha}$ OR more or less right ${ }_{\alpha}$ ),

$\left(\right.$ left $_{\alpha} O R$ very right $\alpha$ ),

$\left(\right.$ left $_{\alpha}$ OR right $\alpha$ ),

(left $t_{\alpha}$ OR more or less right ${ }_{\alpha}$ ),

(more or less left $t_{\alpha} O R$ very right ${ }_{\alpha}$ ),

(more or less left $t_{\alpha} O R$ right $_{\alpha}$ ),

(more or less left $t_{\alpha}$ OR more or less right $\left.\left.{ }_{\alpha}\right)\right\rangle$

$\Leftrightarrow \operatorname{Max}\left\{\left(\right.\right.$ very left $t_{\alpha}$, very right $\left.{ }_{\alpha}\right)$,

(very left ${ }_{\alpha}$, right $\left._{\alpha}\right)$,

(very lef $t_{\alpha}$, more or less right ${ }_{\alpha}$ ),

$\left(\right.$ left $_{\alpha}$, very right $\alpha$ ),

$\left(\right.$ left $t_{\alpha}$, right $\left._{\alpha}\right)$,

(left $t_{\alpha}$, more or less right ${ }_{\alpha}$ ),

(more or less left ${ }_{\alpha}$, very right ${ }_{\alpha}$ ),

(more or less left $t_{\alpha}$, right $\alpha_{\alpha}$ ),

( more or less left $t_{\alpha}$, more or less right $\left.\left.t_{\alpha}\right)\right\}$

$=\operatorname{Max}\left\{[\tilde{L}]_{\alpha},[\tilde{R}]_{\alpha}\right\}$

b) Intersection operation of fuzzy linguistic variables

$[\tilde{L}]_{\alpha} \cap[\tilde{R}]_{\alpha}=\operatorname{Min}\left\{[\tilde{L}]_{\alpha},[\tilde{R}]_{\alpha}\right\}$

Proof:

$[\tilde{L}]_{\alpha} \cap[\tilde{R}]_{\alpha}=[\text { left }]_{\alpha}$ AND $[\text { right }]_{\alpha}$

(Associative Law)

$=(\text { very left }, \text { left }, \text { more or less left })_{\alpha}$

$\mathrm{AND}$ (very right, right, lmore or less right $)_{\alpha}$

$=\left\langle\left(\right.\right.$ very left $t_{\alpha} A N D$ very right $\left.\omega_{\alpha}\right)$,

(very left $t_{\alpha}$ AND right ${ }_{\alpha}$ ),

(very left $\alpha_{\alpha} A N D$ more or less right ${ }_{\alpha}$ ),

(left $t_{\alpha}$ AND very right ${ }_{\alpha}$ ),

$\left(\right.$ left $_{\alpha}$ AND right $\left.{ }_{\alpha}\right)$,

(left $t_{\alpha} A N D$ more or less right ${ }_{\alpha}$ ),

(more or less left $t_{\alpha} A N D$ very right $\alpha$ ),

(more or less left $t_{\alpha} A N D$ right $_{\alpha}$ ),

(more or less left $\alpha_{\alpha} A N D$ more or less right $\left.\left.{ }_{\alpha}\right)\right\rangle$ 


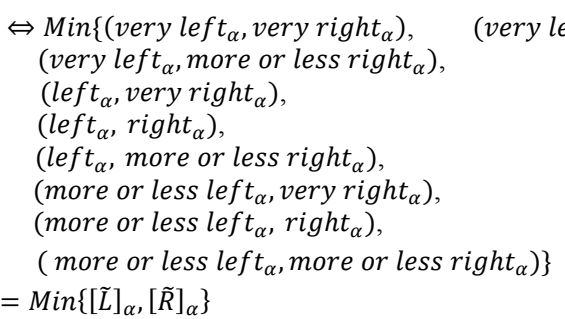

But in linguistic fuzzy geometric modeling discussed only take into account the operation of intersection only.

Proposition 5: Suppose $[\tilde{L}]_{\alpha}$ and $[\tilde{R}]_{\alpha}$ are two fuzzy linguistic variables left and right on the $x$-axis. Operation of intersection $[\tilde{L}]_{\alpha}$ and $[\tilde{R}]_{\alpha}$, will produce three possible cases that may occur and involve linguistic distance.

Definition 6: (Linguistics Distance) Suppose $[\tilde{L}]_{\alpha}$ and $[\tilde{R}]_{\alpha}$ are fuzzy linguistic variables on the same plane and have the terms of linguistics. Metric distance between two linguistic terms $l_{\alpha}(x)$ and $r_{\alpha}(x)$ which has a certain value in the form of linguistic $j(x) \in \tilde{J}$ is defined as

$$
\text { Linguistics Distance, }|\tilde{J}|=\left|l_{\alpha}(x)-r_{\alpha}(x)\right|
$$

Case 1: In this case, there are nine possible intersection points of fuzzy linguistic. However, the intersection point does not produce linguistic distance because the intersection $l_{\alpha}(x)$ and $r_{\alpha}(x)$ occur at the same $\alpha$-cut (See Figure 2).

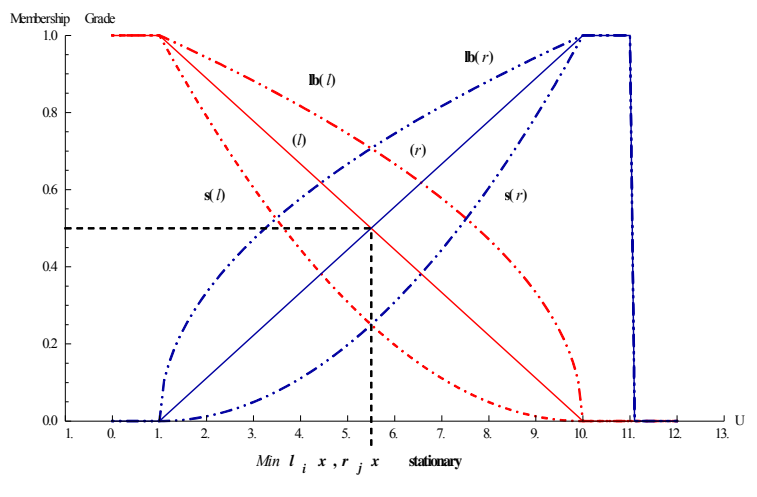

Figure 2. Examples of linguistic variables in case 1

Example 2: left $_{0.5} A N D$ right $_{0.5} \Leftrightarrow \operatorname{Min}\{5,5\}=$ $\operatorname{Min}\left\{\right.$ left $_{0.5}$, right $\left._{0.5}\right\}=$ stationary

Linguistic Distance,

$|\tilde{J}|=\mid$ left $_{0.5}-$ right $_{0.5}|=| 5-5 \mid=0$

Case 2: Suppose that $[\tilde{L}]_{\alpha}$ and $[\tilde{R}]_{\alpha}$ on the different functions of $\alpha$-cut, $l_{\alpha_{i}}(x)$ and $r_{\alpha_{j}}(x)$. If $l_{\alpha_{i}}(x) \neq$ $r_{\alpha_{j}}(x)$ which $\alpha_{i} \neq \alpha_{j} \in[0,1]$, (See Figure 3) then

$\operatorname{Min}\left\{l_{\alpha_{i}}(x), r_{\alpha_{j}}(x)\right\}= \begin{cases}l_{\alpha_{i}}(x) & \text { iff } \alpha_{i}<\alpha_{j} \\ r_{\alpha_{j}}(x) & \text { iff } \alpha_{i} \geq \alpha_{j}\end{cases}$
Linguistic Distance, $|\tilde{J}|=\left|l_{\alpha_{i}}(x)-r_{\alpha_{j}}(x)\right|=|x|$

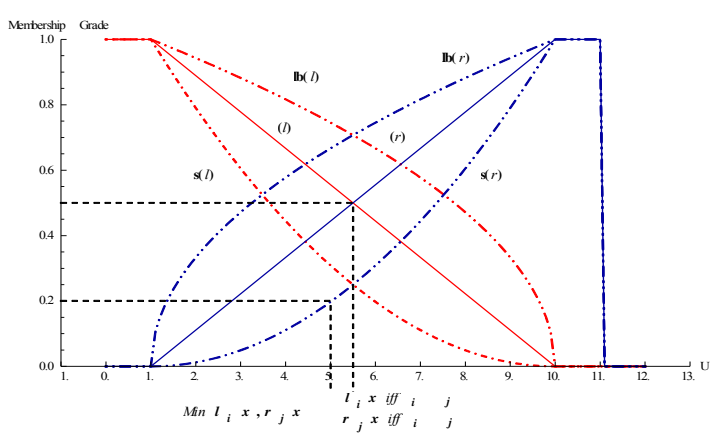

Figure 3. Examples of linguistic variables in case 2

\section{Example 3:}

left $_{0.5} A N D$ very right ${ }_{0.2} \Leftrightarrow \operatorname{Min}\{5,3\}=$ $\operatorname{Min}\left\{\right.$ left $_{0.5}$, very right $\left.{ }_{0.2}\right\}=$ very right Linguistic Distance, $|\tilde{J}|=\mid$ left $_{0.5}-$ very right $_{0.2} \mid=$ $|5-3|=|2|=$ very right

Case 3: Let $[\tilde{L}]_{\alpha}$ and $[\tilde{R}]_{\alpha}$ on the same $\alpha$-cut, $\alpha_{i}=\alpha_{j} \in$ $[0,1]$, but at different functions $l_{\alpha_{i}}(x)$ and $r_{\alpha_{j}}(x)$. If $l_{\alpha_{i}}(x) \neq r_{\alpha_{j}}(x)$, which $\quad \alpha_{i}=\alpha_{j} \in[0,1] \quad$ then $\operatorname{Min}\left\{l_{\alpha_{i}}(x), r_{\alpha_{j}}(x)\right\}$ depending on the linguistic distance (See Figure 4):

$|\tilde{J}|=\left\{\begin{array}{l}\left|l_{\alpha_{i}}(x)-r_{\alpha_{j}}(x)\right|<|5| \approx l_{\alpha_{i}}(x) \\ \left|l_{\alpha_{i}}(x)-r_{\alpha_{j}}(x)\right| \geq|5| \approx r_{\alpha_{j}}(x)\end{array}\right.$

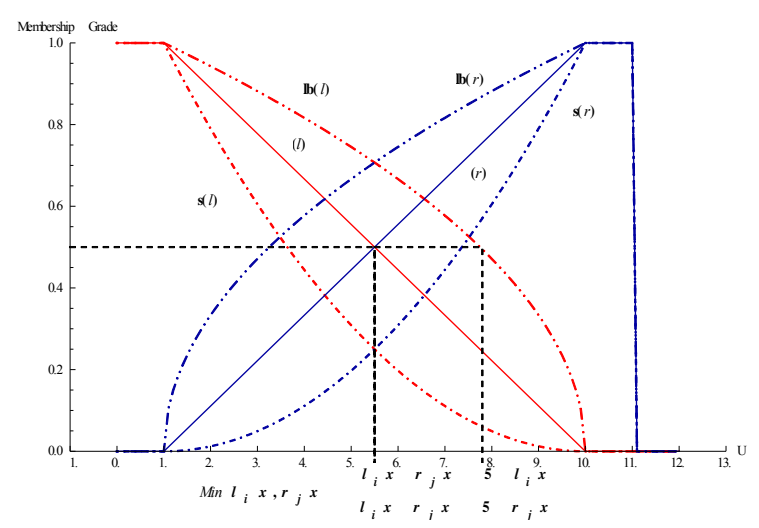

Figure 4. Examples of linguistic variables in case 3

Example 4: left $_{0.5} A N D$ very right ${ }_{0.5} \Leftrightarrow \operatorname{Min}\{5,7\}$

$=\operatorname{Min}\left\{\right.$ left $_{0.5}$, very right v.5 $\left._{0}\right\}$

Linguistic Distance, $|\tilde{J}|$

$=\mid$ left $_{0.5}-$ very right ${ }_{0.5}|=| 5-7|=| 2 \mid<5 \approx$ left

In geometric modeling, usually a control point, $T$ is regarded as the coordinates of point $T(x, y)$ for two dimensions. By this point, based on Theorem $1,[\tilde{A}]_{\alpha}$ and $[\tilde{B}]_{\alpha}$ can be regarded as fuzzy linguistic variables "up" and "down" on the $y$-axis and intersection operations involving fuzzy linguistic variables, such as equations (21) - (26). 
Based on Proposition 5, operation of fuzzy linguistic variables $[\tilde{A}]_{\alpha}$ and $[\tilde{B}]_{\alpha}$ on the $y$-axis will produce three possible cases such as:

Case 4: Intersection operation of fuzzy linguistic point of $a_{\alpha}(y)$ and $b_{\alpha}(y)$ did not produce linguistic distance because the intersection occurs at the same functions and cut- $\alpha$.

Let $[\tilde{A}]_{\alpha}$ and $[\tilde{B}]_{\alpha}$ at the same function of $\alpha$-cut $a_{\alpha_{m}}(y)$ and $b_{\alpha_{n}}(y)$. If $a_{\alpha_{m}}(y)=b_{\alpha_{n}}(y)$, then the $\operatorname{Min}\left\{a_{\alpha_{m}}(y), b_{\alpha_{n}}(y)\right\}=\operatorname{statinary}($ not moving) and the

$|\tilde{J}|=\left|a_{\alpha_{m}}(y)-b_{\alpha_{n}}(y)\right|=0$

\section{Example 5:}

$\operatorname{down}_{0.5} A N D$ up $_{0.5} \Leftrightarrow \operatorname{Min}\{7,7\}=$ $\operatorname{Min}\left\{\right.$ down $\left._{0.5}, u_{0.5}\right\}=$ stationary

Linguistic Distance, $\quad|\tilde{J}|=\mid$ bawah $_{0.5}-$ atas $_{0.5} \mid=$ $|7-7|=0$

Case 5: Suppose $[\tilde{A}]_{\alpha}$ and $[\tilde{B}]_{\alpha}$ at different $\alpha$-cut function $a_{\alpha_{m}}(y)$ and $b_{\alpha_{n}}(y)$. If $a_{\alpha_{m}}(y) \neq b_{\alpha_{n}}(y)$ where $\alpha_{m} \neq \alpha_{n} \in[0,1]$ then

$\operatorname{Min}\left\{a_{\alpha_{m}}(y), b_{\alpha_{n}}(y)\right\}=\left\{\begin{array}{l}a_{\alpha_{m}}(y) \text { iff } \alpha_{m}<\alpha_{n} \\ b_{\alpha_{n}}(y) \text { iff } \alpha_{m} \geq \alpha_{n}\end{array}\right.$

Linguistic Distance, $|\tilde{J}|=\left|a_{\alpha_{m}}(y)-b_{\alpha_{n}}(y)\right|=|y|$

\section{Example 6:}

down $_{0.5} A N D$ very $u p_{0.3} \Leftrightarrow \operatorname{Min}\{7,4\}$

$=\operatorname{Min}\left\{\right.$ down $_{0.5}$, very $\left.u p_{0.3}\right\}=$ very up

Linguistic Distance, $|\tilde{J}|=\mid$ down $_{0.5}-$ very $u p_{0.3} \mid=$ $|7-4|=|3|=$ very $u p$

Case 6: Suppose $[\tilde{A}]_{\alpha}$ and $[\tilde{B}]_{\alpha}$ on the same $\alpha$-cut function $\alpha_{m}=\alpha_{n} \in[0,1]$, but at different functions $a_{\alpha_{m}}(y)$ and $b_{\alpha_{n}}(y)$. If $a_{\alpha_{m}}(y) \neq b_{\alpha_{n}}(y)$ where $\alpha_{m}=$ $\alpha_{n} \in[0,1], \quad$ then $\operatorname{Min}\left\{a_{\alpha_{m}}(y), b_{\alpha_{n}}(y)\right\}$ depending on the linguistic distance:

$|\tilde{J}|=\left\{\begin{array}{l}\left|a_{\alpha_{m}}(y)-b_{\alpha_{n}}(y)\right|<|5| \approx a_{\alpha_{m}}(y \\ \left|a_{\alpha_{m}}(y)-b_{\alpha_{n}}(y)\right| \geq|5| \approx b_{\alpha_{n}}(y)\end{array}\right.$

\section{Example 7:}

down $_{0.5} A N D$ very $u p_{0.5} \Leftrightarrow \operatorname{Min}\{7,10\}=$

$\operatorname{Min}\left\{\right.$ down $_{0.5}$, very $\left.\mathrm{up}_{0.5}\right\}$

Linguistic Distance, $|\tilde{J}|$

$$
\begin{gathered}
=\mid \text { down }_{0.5}-\text { very up } \\
\\
\approx \text { down }
\end{gathered}
$$

\section{FUZZY LINGUISTIC BEZIER MODEL}

In geometric modeling, a control points as a geometry coefficient to change the shape of spline curve and surface without affecting the overall shape of the model. However, in this model, the control points are considered as fuzzy linguistic variables corresponding to the coordinates of $T(x, y)$. Obtained control points are represented by specific linguistic terms that fit in real space of $x$-axis and $y$-axis. By this point, the conventional control points is given the new definition using fuzzy linguistic approach.

Definition 8: Let $\tilde{x}=\left(\operatorname{Min}\left\{l_{\alpha_{i}}(x), r_{\alpha_{j}}(x)\right\}\right)$ is a linguistic point on the $x$-axis and $\tilde{y}=$ $\left(\operatorname{Min}\left\{b_{\alpha_{i}}(y), a_{\alpha_{j}}(y)\right\}\right)$ is a linguistic point on the $y$ axis. If $\tilde{x}$ and $\tilde{y}$ are plotted on a real space $X \times Y$, then $(\tilde{x}, \tilde{y})$ called Fuzzy Linguistics Coordinates.

Definition 9: Let $T=\left\{T_{i}\right\}_{i=0}^{n}$ is a set of control points in space $S$ where $S \in(x, y) . \quad\left[\tilde{T}_{i}\right] \in \tilde{T}$ is said Fuzzy Linguistic Control Point (FLCP) where $\left[\tilde{T}_{i}\right]$ has a fuzzy modifier on $\alpha$-cut, $\alpha \in[0,1] .\left[\tilde{T}_{i}\right]=\tilde{T}\left(\tilde{x}_{i}, \tilde{y}_{i}\right)$ where $\left(\tilde{x}_{i}, \tilde{y}_{i}\right)$ is the Fuzzy Linguistics Coordinates at $i=$ $0,1,2, \ldots, n$

Fuzzy Linguistic Control Points can be rewritten as

$\tilde{T}\left(\tilde{x}_{i}, \tilde{y}_{i}\right)=\left(\operatorname{Min}\left\{l_{\alpha_{i}}(x), r_{\alpha_{j}}(x)\right\}, \operatorname{Min}\left\{b_{\alpha_{i}}(y), a_{\alpha_{j}}(y)\right\}\right)$

If FLCP blended with spline basis functions, the number of Fuzzy Linguistic Spline model can be produced. However, this paper only discusses FLCP that blended with Bezier basis functions.

Definition 10: Assume that Fuzzy Linguistic Control Points $\tilde{T}$ were blended with the Bezier basis functions; a model of Fuzzy Linguistic Bezier curves can be generated:

$\tilde{B}_{F L}(t)=\sum_{i=0}^{n} \tilde{T}_{i} B_{i}^{n}(t)$ with $0 \leq t \leq 1$

where $\tilde{T}_{i}$ is FLCP and $B_{i}^{n}(t)=\left(\begin{array}{l}n \\ i\end{array}\right) t^{i}(1-t)^{n-i}$ is a Bernstein polynomial with degree $n$.

\section{DISCUSSION AND NUMERICAL EXAMPLE}

In this section we will discuss some numerical examples of Fuzzy Linguistics Bezier model (FLB). Bezier curves are generated through linguistic command appearing on Fuzzy Linguistic Control Points. For example, Figure 5 shows three curves of Fuzzy Linguistics Bezier and a conventional Bezier curve (dashed) generated by four control points. Fuzzy Linguistic Bezier curves in the figure are very left and very down with different degrees of membership. The second control points of $\tilde{T}_{1}$ is the control points of a fuzzy membership degree in linguistics with varying the role change the shape of the curve by using linguistic. Bezier curve in the upper layers produced by Fuzzy Linguistic Control Points very left and very down with the membership degree at $\alpha=0.2$. The next curve of Fuzzy Linguistic Bezier curve with membership degree 
at $\alpha=0.5$, followed by the curve at $\alpha=0.8$ degrees of membership. Linguistic Fuzzy control points can be rewritten as

$\tilde{T}_{1}^{0.8}=\tilde{T}_{1}$ (very left $t_{0.8}$, very down $n_{0.8}$ ),

$\tilde{T}_{1}^{0.5}=\tilde{T}_{1}$ (very left $t_{0.5}$, very down $n_{0.5}$ ),

$\tilde{T}_{1}^{0.2}=\tilde{T}_{1}\left(\right.$ very left $t_{0.2}$, very down $n_{0.2}$ )

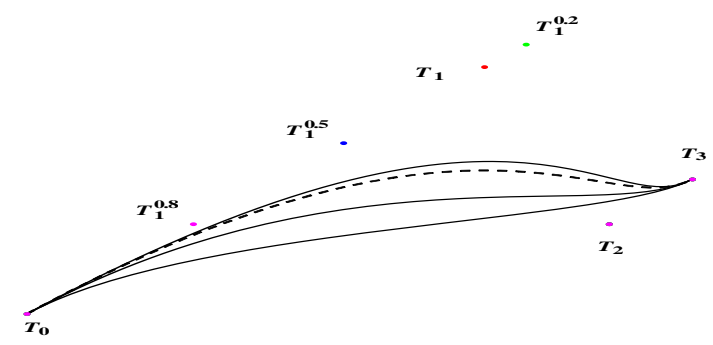

Figure 5. Fuzzy Linguistic Bezier curves model with very left and the very down with the different degree of membership

Figure 6 shows the Bezier curve generated through seven control points $T_{i}=$ $\left\{T_{0}, T_{1}, \widetilde{T}_{2}, T_{3}, \widetilde{T}_{4}, T_{5}, T_{6}\right\}$. Based on the figure, clearly seen that the third control point, $\widetilde{T}_{2}$ and the fifth, $\widetilde{T}_{4}$ is Fuzzy Linguistic Control Point. Curve (dashed) is the conventional Bezier curve and otherwise is Fuzzy Linguistics Bezier with very left at the membership degree $\alpha=0.2, \alpha=0.3$ and $\alpha=0.5$. Fuzzy Linguistic Control Points $\widetilde{T}_{2}$ and $\widetilde{T}_{4}$ respectively can be written as

$\tilde{T}_{2}^{0.2}=\tilde{T}_{2}\left(\right.$ very left $\left.t_{0.2}\right), \ldots, \tilde{T}_{4}^{0.2}=\widetilde{T}_{4}\left(\right.$ very left $\left.t_{0.2}\right)$

$\widetilde{T}_{2}^{0.3}=\widetilde{T}_{2}\left(\right.$ very left $\left.t_{0.3}\right), \ldots, \widetilde{T}_{4}^{0.3}=\widetilde{T}_{4}\left(\right.$ very left $\left.t_{0.3}\right)$

$\tilde{T}_{2}^{0.5}=\tilde{T}_{2}\left(\right.$ very left $\left.t_{0.5}\right), \ldots, \tilde{T}_{4}^{0.5}=\tilde{T}_{4}\left(\right.$ very left $\left.t_{0.5}\right)$

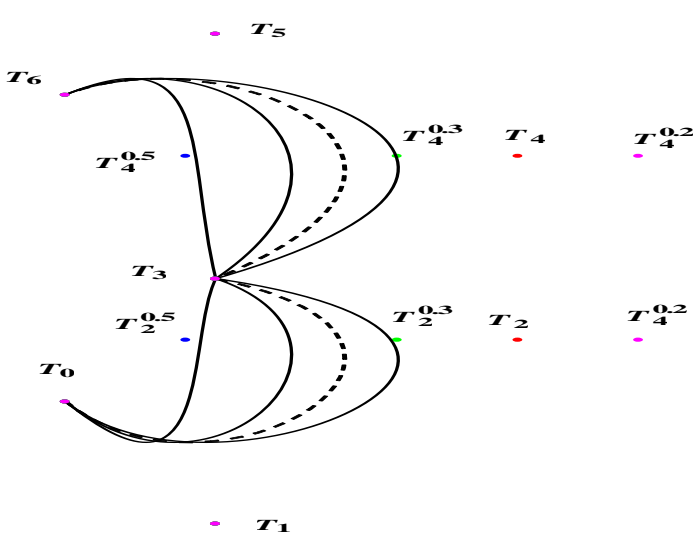

Figure 6. Fuzzy Linguistic Bezier curves model with very left at the degree of membership $\alpha=0.2, \alpha=0.3$ and $\alpha=0.5$

\section{CONCLUSION}

This paper discusses a new concept of fuzzy geometric modeling by introducing Fuzzy Linguistic Control Point (FLCP). The control points is a geometric coefficient linguistically and capable of producing fuzzy spline curve model that is characterized by linguistic. The model presented can be used in other spline models such as B-spline and NURBS. Moreover, this model can also be developed in the design of log-aesthetic curve $[14,20]$.

\section{ACKNOWLEDGEMENTS}

On this occasion, we would like to give our sincere appreciation to the Research Management Centre (RMC), Universiti Malaysia Terengganu and the Ministry of Education Malaysia for research grant ERGS (Vote 55 099) to conduct this research.

\section{References}

[1] A. Bansal. International Journal of Physical and Mathemtical Sciences. (2011). 39-44.

[2] A. F. Wahab, J. M.Ali, A. A. Majid, and A. O. M.Tap, Proceedings International Conference on Computer Graphics, Imaging and Visualization (CGIV04) IEEE Computer Society, (2004); pp. 227-232.

[3] A. F. Wahab, J. M. Ali, A.O.M. Tap and A. A. Majid, LUMS International of Mathematical Modeling and IT. 5-7 November, 2005, Lahore University of Management Sciences (LUMS). (2005)

[4] A. F. Wahab, J. M.Ali, A. A. Majid andA. O. M.Tap,. $6^{\text {th }}$ International Conference on Computer Graphics, Imaging and Visualization, CGIV, 11-14 August 2009, Tianjin, China, (2009);pp.227-232.

[5] A. F. Wahab \& M. H. Sallehuddin. European Journal of Scientific Research. (2011). 272-278.

[6] A. Chandramohan and M. V. C. Rao. Discrete Dynamics in Nature and Society, (2006).

[7] A. M. Anile, B. Falcidieno, G. Gallo, M. Spagnuolo, and S. Spinello. Fuzzy Sets Syst, (2000) 113(3), pp.397-410.

[8] B. Bouchon IEEE International Conference on Fuzzy Systems. (1992).247-254

[9] D. C. Martine, \& K.E. Etienne. Information Sciences, (2004), 160(1-4), pp.173-199.

[10] D. Dubois \& H. Prade. Academic Press. (1980).

[11] F. Eshragh \& E. H. Mamdani. Academic Press. (1981).

[12] G. Gallo, M. Spagnuolo and S. Spinello, Journal of Geographic Information and Decision Analysis. (1998), 2(2): pp.194-203.

[13] G. Gallo, S. Spagnuolo and S. Spinello, Graphical Models. (2000), 62: pp.40-55.

[14] K. T. Miura, Dai Shibuya, R.U. Gobithaasan \& Shin Uzuki, Computer Aided Design \& Application, (2013). Vol.10(6), 1021-1032.

[15] L. A. Zadeh, Information and Control. (1965).(8)338-353.

[16] L. A. Zadeh, Journal of Cybernetics, (1972), 2(3), pp.4-34.

[17] L. A. Zadeh. Information Sciences. (1975). (8)199-249.

[18] L. D. Lascio, A. Gisolfi and V. Loia. International Journal of Approximate Reasoning. (1996), (15)25-47.

[19] M.H. Sallehuddin, and A. F. WahabUniversity Publication Centre (UPENA), UiTM 2010. (2010), pp. 21-28.

[20] R. U. Gobithaasan and K. T. Miura, , Sains Malaysiana (2011), 40 (11), pp.1301-1305.

[21] T. Hajjari. ICMS International Conference on Mathematical Science. (2010). 346-356.

[22] T. Zamali, M. A. Lazim \& M. T. Abu Osman. IEEE Symposium on Humanities, Science and Engineering Research. (2012).669672.

[23] V. N. Huynh, T. B. Ho and Y. Nakamori, International Journal of Approximate Reasoning, (2002), 30(3), pp.203-223.

[24] Z. Rozaimi and A. F. Wahab, University Publication Centre (UPENA), UiTM2010. (2010); pp. 53-60. 